\title{
FUZZY OPTIMIZATION OF COST FUNCTION IN PRODUCT MIX SELECTION PROBLEM
}

\author{
${ }^{1}$ Pandian M. Vasant, ${ }^{2}$ Nadir N. Barsoum \\ ${ }^{1}$ Electrical \& Electronic Engineering Program, Universiti Teknologi Petronas, Malaysia \\ E-mail: pandian_m@petronas.com.my,pandian_vasant@yahoo.com \\ ${ }^{2}$ School of Electrical \& Electronic Engineering, Curtin University, Malaysia \\ E-mail: nnb3@hotmail.com
}

\begin{abstract}
The modern trend in industrial application problem deserves modeling of all relevant vague or fuzzy information involved in a real decision making problem. In this paper the usefulness of the proposed S-curve membership function is established using a real life industrial production planning of a chocolate manufacturing unit. The unit produces 8 products using 8 raw materials; mixed in various proportions by 9 different processes under 29 constraints. A solution to this problem establishes the usefulness of the suggested membership function for decision making in industrial production planning. The objective of this paper is to find the optimal cost to produce 8 products using modified Scurve membership function as a methodology. The fuzzy linear programming approach is used to solved this problem. The optimal cost function is obtained respect to two major factors of degree of satisfaction and vagueness. Copyright $\odot 2005$ IFAC
\end{abstract}

Keywords: Decision making, fuzzy systems, fuzzy linear programming, product-mix selection, optimization

\section{INTRODUCTION}

Due to limitations in resources for manufacturing a product and the need to satisfy certain conditions in manufacturing and demand, a problem of fuzziness occurs in industrial production planning. This problem occurs also in chocolate manufacturing when deciding a mixed selection of raw materials to produce varieties of products. This is referred here to as the Product- mix Selection Problem (Tabucanon, 1996). The data for this problem are taken from the data-bank of Chocoman Inc, USA (Tabucanon, 1996). Chocoman produces varieties of chocolate bars, candy and wafer using a number of raw materials and processes. The objective is to use the modified S-shaped membership function for obtaining a cost optimization procedure through FLP (Fuzzy Linear Programming).
The modified S-curve membership function is proved to be a flexible membership function through an analytical approach (Vasant, 2002). This membership function is to be used in FLP involving fuzzy objective coefficients, fuzzy technical coefficients and fuzzy resource variables. The modified S-curve membership function is flexible enough to describe vagueness in these fuzzy parameters.

The objective of the company is to minimize its cost, which is, alternatively, equivalent to minimizing the cost to produce eight products. That is to find the optimal product mix under uncertain constraints in the technical, raw material and market_consideration. Furthermore, it is possible to show the relationship between the optimal cost and the corresponding membership values (Vasant et al., 2002a). According 
to this relationship, the decision maker can then obtain his optimal solution with a trade-off under a pre-determined allowable imprecision (Zimmermann, 1985) and (Vasant and Barsoum, 2005).

\section{METHODOLOGY FOR FPS PROBLEM}

The Fuzzy Product - mix Selection Problem (FPS) is stated as:

There are eight products to be manufactured by mixing eight raw materials with different proportion and by using nine varieties of processing. There are limitations in resources of raw materials. There are also ten constraints imposed by marketing department such as product - mix requirement, main product line requirement and lower and upper limit of demand for each product. All the above requirements and conditions are fuzzy. It is necessary to obtain optimal cost with certain degree of satisfaction by using fuzzy linear programming and modified S-curve membership function.

The first step is construction of S-curve membership function for the FPS problem. Then followed by formulation of FLP problem which represent FPS problem. This mathematical model of FLP problem will be solved by using LP toolbox in MATLAB ${ }^{\circledR}$.

Here we only consider one problem of FPS in which the objective coefficients, technical coefficients and resource variables all are fuzzy. The FLP model for this problem is given in (1). The objective function is the cost for the FPS problem.

$$
\begin{array}{ll}
\text { Minimize } & z=\sum_{j=1}^{8}{\stackrel{c}{j} x_{j}} \\
\text { subject to } & \sum_{i=1}^{29} a_{i j} \leq b_{i}
\end{array}
$$

where $\tilde{c_{j}}, \tilde{a}_{i j}$ and $\tilde{c_{j}}$ are fuzzy parameters.

The relation between objective coefficient of cost function and vagueness is given in the following equation.

$$
\left.\widetilde{c}_{j}\right|_{\mu=\mu_{C_{J}}}=c_{j}^{a}+\left(\frac{c_{j}^{b}-c_{j}^{a}}{\alpha}\right) \ln \frac{1}{C}\left(\frac{B}{\mu_{c_{j}}}-1\right)
$$

The following values are substituted in the equation (2) with $C=0.001001001, \mathrm{~B}=1, \alpha>0$ and $\alpha=$ 13.8135 (Vasant, 2002).

Equation(1) is solved by using parametric programming approach (Carlsson and Korhonen, 1986) and a modified S-curve membership function used as a methodology (Vasant et al., 2002b;
Vasant 2004). The input data for $c_{j}$ is the cost fuzzy values, $a_{i j}$ technical coefficients and $b_{i}$ is the resource variables for FPS problem. The fuzzy interval for objective coefficients $c_{j}$ (cost) is calculated from the profit and revenue optimization (Tabucanon, 1996). The interval for cost is selected such a way that it's value less than profit. There are 29 constraints and 8 products and hence in (1), i = 1, 2, 3,...,29 and $\mathrm{j}=$ $1,2,3, \ldots, 8$. Membership function and membership values for $C_{j}$ 's are constructed and valued. The FLP problem has been formulated and all the coefficients are parameterized. However, it will not be possible to use the linear parametric formulation to solve the FLP problem since the membership functions are non-linear (Watada, 1997). Then, it is needed to carry out a series of experiments for 21 membership values: $\mu_{\mathrm{aij}}=\mu_{\mathrm{bi}}=\mu_{\mathrm{cj}}=\mu=0.0010,0.0509$, $0.1008, \ldots, 0.9990$ with an interval of 0.0499 . These experiments are carried out by using the Simplex Method in the Optimization Tool Box of MATLAB ${ }^{\circledR}$.

First of all, construct the membership functions for the fuzzy parameters of $\tilde{A}, \tilde{c}_{j}$ and $\tilde{b}$. Here a nonlinear membership function called as modified Scurve membership function is used for the convenience of selecting vague parameter $\alpha$ (Bells, 1999 and Kuzmin, 1981). The membership functions are represented by $\mu_{a_{i j}}$, and $\mu_{b_{i}}$, where $a_{i j}$ are the technical coefficients of matrix $A$ for $i=1, \ldots, 29$ and $j=1, \ldots, 8, b_{i}$ are the resource variables for $i=1, \ldots, 29$. The membership function for $\mu_{a_{i j}}$ and the fuzzy interval, $a_{i j}^{a}$ to $a_{i j}^{b}$, for $\tilde{a}_{i j}$ is given in Vasant (2004).

In similar way we can construct membership function for fuzzy resource variable and fuzzy objective coefficient and it's derivations (Vasant, 2002). Since the technical coefficients and resource variables are fuzzy therefore the outcome of the cost function will be fuzzy.

\section{FUZZY SOLUTIONS AND THE OUTCOME}

The FPS problem is solved by using MATLAB and its toolbox of Linear Programming (LP). The vagueness is given by $\alpha$, and $\mu$ is the degree of satisfaction. The LP toolbox has two inputs namely $\alpha$ and $\mu$ in addition to the fuzzy parameters. There is one output $z^{*}$, the optimal cost function.

The given values of various parameters of Chocolate Manufacturing are fed to the toolbox. The solution can be tabulated and presented as 2 and 3 dimensional graphs. 


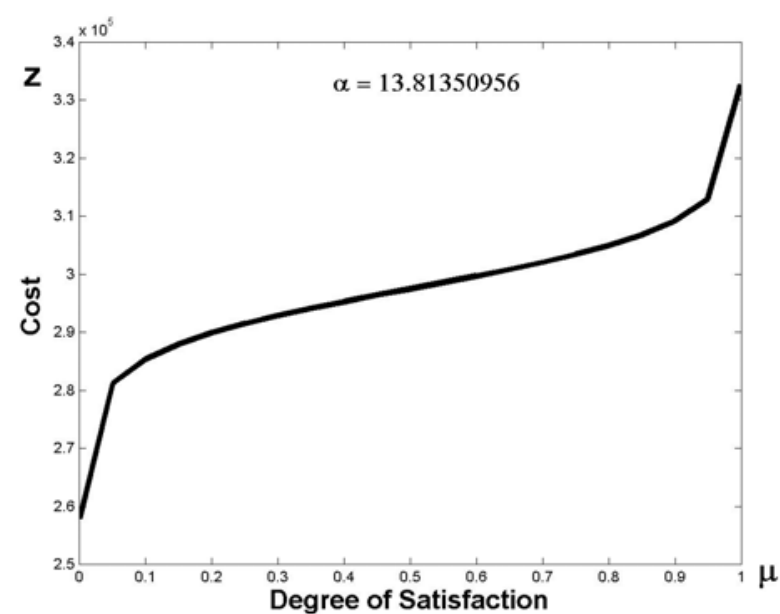

Figure II- Optimal Cost and Degree of Satisfaction $(\alpha=13 . .8135)$

Table 1- Optimal Cost and Degree of Satisfaction ( $\alpha=13 . .8135)$

\begin{tabular}{|c|c|c|}
\hline No & Degree of Satisfaction $(\mu)$ & $\begin{array}{c}\text { Optimal Cost } \\
\left(\mathrm{z}^{*}\right)\end{array}$ \\
\hline 1 & 0.0010 & 257920 \\
\hline 2 & 0.0509 & 281240 \\
\hline 3 & 0.1008 & 285410 \\
\hline 4 & 0.1507 & 287980 \\
\hline 5 & 0.2006 & 289910 \\
\hline 6 & 0.2505 & 291500 \\
\hline 7 & 0.3004 & 292880 \\
\hline 8 & 0.3503 & 294130 \\
\hline 9 & 0.4002 & 295300 \\
\hline 10 & 0.4501 & 296410 \\
\hline 11 & 0.5000 & 297500 \\
\hline 12 & 0.5499 & 298580 \\
\hline 13 & 0.5998 & 299690 \\
\hline 14 & 0.6497 & 300830 \\
\hline 15 & 0.6996 & 302050 \\
\hline 16 & 0.7495 & 303390 \\
\hline 17 & 0.7994 & 304910 \\
\hline 18 & 0.8493 & 306740 \\
\hline 19 & 0.8992 & 309150 \\
\hline 20 & 0.9491 & 312980 \\
\hline 21 & 0.9990 & 332660 \\
\hline
\end{tabular}

From Figure II, we can see that the graph behaves as an increasing function. This shows that the objective values are increases as degree of satisfaction increases. The cost function (objective value) has a value 332660 at $\mu=0.999$. We define this as $99.9 \%$ degree of satisfaction. Accordingly a $\mathrm{z}^{*}$ of value 257920 has $0.1 \%$ degree of satisfaction. The possible realistic solution exists at $\mu=0.5$ (ie $50 \%$ degree of satisfaction) with a value of $z^{*}$ as 297500 . The nonfuzzy situation (i.e all the coefficients $a_{i j}, c_{j}$ and $b_{i}$ are precise) and the $\mathrm{z}^{*}$ value has been computed to be less than 257920 (Tabucanon, 1996). It is found that $\mathrm{z}^{*}$ becoming more than that of a totally non-fuzzy situation (Vasant, 2002;2003). The result obtained by using fuzzy optimization approach far better than the result obtained by deterministic approach (Tabucanon, 1996). The comparison for the profit function is available in Vasant (2002).

3.1 A. Objective Values for Various $\alpha$
Figure II illustrates the variation of objective values $\mathrm{z}^{*}$ with respect to degree of satisfaction $\mu$ for one value of vagueness factor $\alpha=13.8135$. It will be useful for the decision maker to observe such variations for several values of $\alpha$.

Figure III, shows the nature of variations of $\mathrm{z}^{*}$ with respect to $\mu$ when $\alpha$ varies from 1.5 to 39.5.

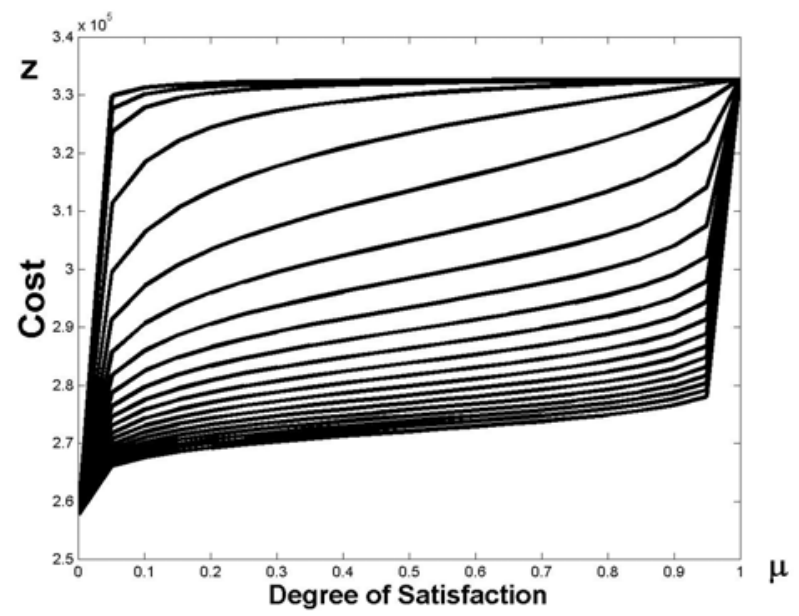

Figure III - Variation of Cost $z^{*}$ in Terms of $\mu$ and $\alpha$

The realistic solution with an uncertainties in fuzzy parameters of technical coefficients and resource variables exists at $\mu=50 \%$. Hence the result for $50 \%$ degree of satisfaction for $1.5 \leq \alpha \leq 39.5$ and the corresponding values for $\mathrm{z}^{*}$ are tabulated in Table 2 .

\begin{tabular}{cc} 
Table 2- Vagueness $\alpha$ and Cost $\mathrm{z}^{*}$ for $\mu$ & $\mu=50 \%$ \\
\cline { 2 - 3 } Vagueness $\alpha$ & ${\text { Cost } \mathrm{z}^{*}}^{\text {Vagu }}$ \\
\hline 1.5 & 332510 \\
3.5 & 332070 \\
5.5 & 330060 \\
7.5 & 323470 \\
9.5 & 313530 \\
11.5 & 304890 \\
13.5 & 298370 \\
15.5 & 293400 \\
17.5 & 289510 \\
19.5 & 286390 \\
21.5 & 283830 \\
23.5 & 281690 \\
25.5 & 279880 \\
27.5 & 278320 \\
29.5 & 276970 \\
31.5 & 275790 \\
33.5 & 274740 \\
35.5 & 273800 \\
37.5 & 272960 \\
39.5 & 272090 \\
\hline
\end{tabular}

The fuzzy outcome for the cost function decreases as vagueness $\alpha$ increases in the fuzzy parameters of technical coefficients, objective coefficients and resource variables. This is clearly shown in Table 2. Table 2 is very important to the decision maker in picking up the $\alpha$ so that the outcome will be at good enough satisfactory level. 
The outcome in Table 3 shows that when the vagueness in increases results in less cost. Also it is found that the S-curve membership function with various values of $\alpha$ provides a possible solution with certain degree of satisfaction.

Furthermore the relationship between $\mathrm{z}^{*}, \mu$ and $\alpha$ is given in Table 3 . This Table is very useful for the decision maker to find the cost any given value of $\alpha$ with degree of satisfaction $\mu$. From Table 3 it is observed that at any particular degree of satisfaction $\mu$ the cost of products $z^{*}$ decreases as the vagueness $\alpha$ increases between 1.5 and 39.5. Similarly at any particular value of vagueness the cost of products are increases as the degree of satisfaction increases.

\begin{tabular}{ccccc}
\multicolumn{5}{c}{ Table 3(a): Fuzzy Optimal Cost for $1.5 \leq \alpha \leq 7.5$} \\
\hline $\mathrm{z}^{*}$ & \multicolumn{4}{c}{ Vagueness $\alpha$} \\
\hline$\mu$ & 1.5 & 3.5 & 5.5 \\
0.0010 & 257920 & 257920 & 257920 & 257920 \\
0.0509 & 329900 & 323710 & 311310 & 299520 \\
0.1008 & 331330 & 327900 & 318470 & 306450 \\
0.1507 & 331810 & 329530 & 322130 & 310560 \\
0.2006 & 332060 & 330400 & 324480 & 313510 \\
0.2505 & 332210 & 330940 & 326050 & 315840 \\
0.3004 & 332310 & 331310 & 327250 & 317780 \\
0.3503 & 332380 & 331580 & 328180 & 319450 \\
0.4002 & 332240 & 331780 & 328930 & 320920 \\
0.4501 & 332480 & 331940 & 329540 & 322250 \\
0.5000 & 332510 & 332070 & 330060 & 323470 \\
0.5499 & 332540 & 332180 & 330490 & 324600 \\
0.5998 & 332560 & 332270 & 330870 & 325660 \\
0.6497 & 332580 & 332340 & 331190 & 326650 \\
0.6996 & 332600 & 332410 & 331480 & 327600 \\
0.7495 & 332610 & 332460 & 331730 & 328510 \\
0.7994 & 332630 & 332510 & 331960 & 329390 \\
0.8493 & 332640 & 332560 & 332160 & 330240 \\
0.8992 & 332650 & 332600 & 332350 & 331060 \\
0.9491 & 332660 & 332630 & 332510 & 338700 \\
0.9990 & 332660 & 332660 & 332660 & 332660
\end{tabular}

Table 3(b): Fuzzy Optimal Cost for $9.5 \leq \alpha \leq 11.5$

\begin{tabular}{ccccc}
\hline $\mathrm{z}^{*}$ & \multicolumn{4}{c}{ Vagueness $\alpha$} \\
\hline$\mu$ & 9.5 & 11.5 & 13.5 & 15.5 \\
0.0010 & 257920 & 257920 & 257920 & 257920 \\
0.0509 & 291340 & 285760 & 281760 & 278770 \\
0.1008 & 297180 & 290700 & 286020 & 282510 \\
0.1507 & 300750 & 293740 & 288650 & 284880 \\
0.2006 & 303410 & 296010 & 290620 & 286560 \\
0.2505 & 305570 & 297870 & 292240 & 287990 \\
0.3004 & 307440 & 299500 & 293650 & 289230 \\
0.3503 & 309110 & 300960 & 294930 & 290360 \\
0.4002 & 310660 & 302320 & 296120 & 291410 \\
0.4501 & 312120 & 303620 & 297260 & 292480 \\
0.5000 & 313530 & 304890 & 298370 & 293400 \\
0.5499 & 314910 & 306150 & 299470 & 294380 \\
0.5998 & 316280 & 307430 & 300600 & 295380 \\
0.6497 & 317680 & 308750 & 301770 & 296410 \\
0.6996 & 319130 & 310150 & 303010 & 297520 \\
0.7495 & 320660 & 311670 & 304370 & 298730 \\
0.7994 & 323310 & 313400 & 305920 & 300110 \\
0.8493 & 324160 & 315440 & 307790 & 301780 \\
0.8992 & 326300 & 318070 & 310230 & 303970 \\
0.9491 & 328970 & 322030 & 314110 & 307480 \\
0.9990 & 332660 & 332660 & 332660 & 332660 \\
\hline
\end{tabular}

Table 3(c): Fuzzy Optimal Cost for $17.5 \leq \alpha \leq 23.5$

\begin{tabular}{ccccc}
\hline $\mathrm{z}^{*}$ & \multicolumn{4}{c}{ Vagueness $\alpha$} \\
\hline$\mu$ & 17.5 & 19.5 & 21.5 & 23.5 \\
0.0010 & 257920 & 257920 & 257920 & 257920 \\
0.0509 & 276440 & 274580 & 273060 & 271790 \\
0.1008 & 279780 & 277590 & 275800 & 274310 \\
0.1507 & 281840 & 279450 & 277500 & 275870 \\
0.2006 & 283390 & 280850 & 278770 & 277040 \\
0.2505 & 284670 & 282010 & 279830 & 278010 \\
0.3004 & 285780 & 283010 & 280750 & 278850 \\
0.3503 & 286790 & 283930 & 281580 & 279620 \\
0.4002 & 287730 & 284780 & 282360 & 280330 \\
0.4501 & 288630 & 285590 & 283100 & 281020 \\
0.5000 & 289510 & 286390 & 283830 & 281690 \\
0.5499 & 290390 & 287190 & 284550 & 282360 \\
0.5998 & 291280 & 288000 & 285300 & 283040 \\
0.6497 & 292210 & 288840 & 286070 & 283750 \\
0.6996 & 293210 & 289740 & 286890 & 284510 \\
0.7495 & 294290 & 290720 & 287790 & 285340 \\
0.7994 & 295540 & 291850 & 288820 & 286290 \\
0.8493 & 297030 & 293210 & 290060 & 287430 \\
0.8992 & 299010 & 295010 & 291710 & 288950 \\
0.9491 & 302180 & 297890 & 294350 & 291390 \\
0.9990 & 332660 & 332660 & 332660 & 332660 \\
\hline & & & &
\end{tabular}

\begin{tabular}{ccccc}
\hline $\mathrm{z}^{*}$ & \multicolumn{4}{c}{ Vagueness $\alpha$} \\
\hline$\mu$ & 25.5 & 27.5 & 29.5 & 31.5 \\
0.0010 & 257920 & 257920 & 257920 & 257920 \\
0.0509 & 270720 & 269800 & 269010 & 268310 \\
0.1008 & 273050 & 271960 & 271030 & 270210 \\
0.1507 & 274490 & 273310 & 272280 & 271380 \\
0.2006 & 275570 & 274320 & 273220 & 272270 \\
0.2505 & 276470 & 275150 & 274000 & 273000 \\
0.3004 & 277250 & 275880 & 274680 & 273640 \\
0.3503 & 277960 & 276530 & 275300 & 274220 \\
0.4002 & 278620 & 277150 & 275880 & 274760 \\
0.4501 & 279260 & 277740 & 276430 & 275280 \\
0.5000 & 279880 & 278320 & 276970 & 275790 \\
0.5499 & 280490 & 278900 & 277510 & 276290 \\
0.5998 & 281130 & 279480 & 278060 & 276810 \\
0.6497 & 281780 & 280100 & 278630 & 277350 \\
0.6996 & 282480 & 280750 & 279240 & 277920 \\
0.7495 & 283250 & 281470 & 279910 & 278550 \\
0.7994 & 284140 & 282290 & 280680 & 279270 \\
0.8493 & 285200 & 283280 & 281610 & 280150 \\
0.8992 & 286600 & 284590 & 282840 & 283160 \\
0.9491 & 288870 & 286700 & 284810 & 283160 \\
0.9990 & 332660 & 332660 & 332660 & 332660 \\
\hline
\end{tabular}

Table 3(e) : Fuzzy Optimal Cost for $33.5 \leq \alpha \leq 39.5$

\begin{tabular}{ccccc}
\hline $\mathrm{z}^{*}$ & \multicolumn{4}{c}{ Vagueness $\alpha$} \\
\hline$\mu$ & 33.5 & 35.5 & 37.5 & 39.5 \\
0.0010 & 257920 & 257910 & 257900 & 257780 \\
0.0509 & 267700 & 267140 & 266640 & 266090 \\
0.1008 & 269480 & 268830 & 268240 & 267610 \\
0.1507 & 270590 & 269880 & 269240 & 268550 \\
0.2006 & 271430 & 270670 & 269990 & 269260 \\
0.2505 & 272120 & 271320 & 270600 & 269850 \\
0.3004 & 272720 & 271890 & 271140 & 270360 \\
0.3503 & 273260 & 272400 & 271630 & 270830 \\
0.4002 & 273770 & 272890 & 272090 & 271260 \\
0.4501 & 274260 & 273350 & 272530 & 271680 \\
0.5000 & 274740 & 273800 & 272960 & 272090 \\
0.5499 & 275220 & 274250 & 273390 & 272500 \\
0.5998 & 275710 & 274710 & 274090 & 272910 \\
0.6497 & 276210 & 275190 & 274280 & 273350 \\
0.6996 & 276750 & 275710 & 274770 & 273810 \\
0.7495 & 277350 & 276270 & 275300 & 274320 \\
0.7994 & 278030 & 276910 & 275910 & 274900 \\
0.8493 & 278850 & 277690 & 276650 & 275600 \\
0.8992 & 279940 & 278730 & 277630 & 276540 \\
0.9491 & 281700 & 280390 & 279210 & 278040 \\
0.9990 & 332660 & 332660 & 332660 & 332660 \\
\hline
\end{tabular}


$\mu=$ Degree of Satisfaction, $z^{*}=$ Cost of Products and $\alpha=$ Vagueness.

The diagonal values in the Tables 3 , show that the cost increases at lower value of $\mu(0.1 \% \leq \mu \leq$ $5.09 \%)$. Then $\mathrm{z}^{*}$ value decreases for $5.09 \%<\mu \leq$ 89.92\%. Lastly $\mathrm{z}^{*}$ value increases for $89.92 \%<\mu \leq$ 99.9\%. This result shows that good decision (higher degree of satisfaction) does not guarantee minimum value in cost (objective value). This means one should satisfy with degree of satisfaction when come to making decision in a fuzzy environment. The result shows that, the outcome almost does not depend on the decision made at early stage of input level for fuzzy parameters of objective coefficients, technical coefficients and resource variables.

\section{CONCLUSION}

The S-curve membership function was used in generating fuzzy parameters towards solving an industrial production-planning problem. These parameters are defined in terms of the fuzzy linear programming problem and named as the fuzzy coefficients of the objective function, fuzzy technical coefficients and fuzzy resource variables. Membership values for this fuzzy parameters are created by using the S-curve membership function. This formulation is found to be suitable in applying the Simplex Method in Linear programming (LP) approach. This approach of solving industrial production planning problem can have feed back within the decision maker, the implementer and the analyst. It is to be noted that minimum cost need not lead to higher degree satisfaction. The decision maker has to satisfy with the cost, which was obtained through FLP process respect to degree of satisfaction. Since the emphasis is given to degree of satisfaction and vagueness in the fuzzy system so the problem of finding the well distributed $\mathrm{z}^{*}$ function becomes very important in this work. In order to obtain this, we need experience and expertise in selecting parameter $\alpha$. The Tables and Figures are very useful for the decision maker and the implementer to make final decision for picking up the optimal cost.The input data for cost function was calculated from profit function and return. Since both profit and return are fuzzy input therefore the cost function also has to be fuzzy. The selection of fuzzy cost function for input data has made such a way that the value is less then the profit. The outcome of the cost function for this FPS problem almost equal to the profit function. It is possible to reduce the cost function by carrying out the solution procedure continuously in an interactive manner between decision maker and analyst. This will result in fuzzy system of industrial production system of interactive process. The best good enough outcome for minimum cost function can be achieved by designing self-organizing fuzzy system for the FPS problem.

\section{ACKNOWLEDGEMENT}

The authors would like to sincerely thank you for the reviewers for their valuable comments and suggestions to improve the final version of the paper.

\section{REFERENCES}

Bells, S.(1999). Flexible Membership Functions. Http:/www. Louder than a bomb com/ spark features. Html.

Carlsson, C and Korhonen, P. A (1986) Parametric Approach to Fuzzy Linear Programming. Fuzzy Sets And System 20, pp. 7-30.

Kuz'min, V. B.(1981). A Parametric Approach to Description Linguistic Values of Variables and Hedges. Fuzzy Sets and Systems , 6, pp. 27-41.

Tabucanon, M. T (1996). Multi Objective Programming for Industrial Engineers. In: Mathematical Programming for Industrial Engineers (M. Avriel and B. Golany), pp. 487542. Marcel Dekker, Inc, New York,

Vasant, P. M (2002) A Methodology of Decision Making in an Industrial Production Planning Using Interactive Fuzzy Linear Programming. M. Sc. Thesis., School of Engineering and Information Technology, University Malaysia Sabah, Malaysia.

Vasant, P. M, Nagarajan, R and Yaacob, S. (2002a). Fuzzy Linear Programming: A Modern Tool For Decision Making, 2002 International Conference on Fuzzy Systems and Knowledge Discovery, Singapore, pp 772-776.

Vasant, P. M, Nagarajan, R and Yaacob, S. (2002b). Fuzzy Mix Product Selection in an Interactive Production Planning, 2002 Annual Meeting of the North American Fuzzy Information Processing Society Proceedings, USA, pp 360 - 364.

Vasant, P. M., (2003). Application of Fuzzy Linear Programming in Production Planning, Fuzzy Optimization and Decision Making, 3, pp. 229241.

Vasant, P. M., (2004). Industrial Production Planning Using Interactive Fuzzy Linear Programming. International Journal of Computational Intelligence and Applications 4, 13-26.

Vasant, P. M and Barsoum, N. N. (2005). Fuzzy Optimization of Units Products in Mix-Products Selection Problem Using FLP Approach, Soft Computing Journal, (to appear).

Watada, J. (1997). Fuzzy Portfolio Selection and Its Applications to Decision Making. Tatra Mountains Mathematics Publication 13, 219-248.

Zimmermman, H. J (1985). Application of Fuzzy Set Theory to Mathematical Programming. Information Sciences, 36, 25-58. 